\title{
Research in brief: Coagulopathy in COVID-19: Determining and managing thrombotic risk in COVID-19 infection
}

\author{
Author: Rajan S Pooni ${ }^{\mathrm{A}}$
}

\section{DOI: $10.7861 /$ clinmed.rib.20.4.2}

\section{Background}

Summary of Stoneham SS, Milne KM, Nuttall E et al. Thrombotic risk in COVID-19: a case series and case-control study. Clin Med 2020;20:e76-81.

The observation of an underlying hypercoagulable state in COVID-19 infection has raised questions about how best to determine and manage its underlying risk in preventing and treating venous thromboembolism (VTE). ${ }^{1}$ The high incidence of VTE in COVID-19 is comparable to other viral pneumonias (notably severe acute respiratory syndrome (SARS) and Middle East respiratory syndrome (MERS-CoV)), particularly in patients with prolonged disease or in multiorgan failure. ${ }^{2,3}$ However, the pathogenesis of hypercoagulability remains unclear. ${ }^{4}$ Current hypotheses include endothelial injury as a direct effect of virus invasion and sepsis-induced coagulopathy (SIC) manifesting as a disseminated intravascular coagulopathy (DIC)-like syndrome, with thrombosis predominating rather than bleeding. ${ }^{1,3,5}$

Existing literature has focused almost solely on VTE in COVID-19 infection in the context of intensive care or high-dependency settings. In this clinical summary we review the association between COVID-19 infection and thrombotic events in ward-based patients admitted to Brighton and Sussex University Hospitals NHS Trust from 20 March 2020 to 09 April 2020.

\section{Study findings}

Two-hundred and seventy-four patients with either confirmed (PCR positive) or probable (radiographic evidence) of COVID-19 underwent biochemical evaluation of laboratory markers, specifically their D-dimer values, to determine whether there was any statistical difference between the group of patients with confirmed VTE (cases) compared those patients without VTE (controls).

Of the 274 patients, 21 (7.7\%) were diagnosed with VTE (pulmonary embolism or deep vein thrombosis) and unsurprisingly this group of patients demonstrated higher $(>0.5 \mu \mathrm{g} / \mathrm{mL}) \mathrm{D}$-dimer values $(4.1 ; p<0.001)$ when compared to the control group. Of note is the confirmation that patients in the control group also demonstrated the hypercoagulable state, with an average D-dimer of 1.2. With such a high risk of VTE observed in the control group,

Author: ${ }^{\mathrm{A}}$ internal medicine trainee, Newham University Hospital, London, UK despite thromboprophylaxis, perhaps there is a role for $\mathrm{D}$-dimer level-guided thromboprophylaxis regimens. ${ }^{6,7}$

Perhaps most interestingly is the identification of a cohort of patients who were refractory to treatment with standard anticoagulation. Three such patients with confirmed VTE demonstrated subtherapeutic anti-factor Xa levels or activated partial thromboplastin time (APTT) ratio despite administration of weight-based low molecular weight or unfractionated heparin. All three patients exhibited very high levels of factor VIII and von Willebrand factor that may represent both a marker of SARS-CoV-2 induced endothelial damage and an important mechanistic cause of COVID-19 related thrombosis.

\section{What does this mean in practice?}

> Hypercoagulability is a recognised state in COVID-19 infection. Markedly elevated D-dimer values should increase clinical suspicion VTE and may warrant further investigation.

> Further haematological investigation should be considered in patients with confirmed VTE, particularly if no clinical improvement is observed and clot progression is suspected.

> Anti-factor Xa levels and APTT ratios are a good way to determine the efficacy of weight-based low molecular weight and unfractionated heparin regimens in patients with confirmed VTE.

\section{References}

1 The Lancet Haematology. COVID-19 coagulopathy: an evolving story. Lancet Haematol 2020;7:e425.

2 Moores LK, Tritschler T, Brosnahan S et al. Prevention, diagnosis and treatment of venous thromboembolism in patients with COVID-19: CHEST Guideline and Expert Panel Report. Chest 2020:S0012-3692(20)31625-1 [Epub ahead of print].

3 Connors JM, Levy JH. COVID-19 and its implications for thrombosis and anticoagulation. Blood 2020;135:2033-40.

4 Iba T, Levy JH, Levi M, Thachil J. Coagulopathy in COVID-19. J Thromb Haemost 2020 [Epub ahead of print].

5 Panigada M, Bottino N, Tagliabue P et al. Hypercoagulability of COVID-19 patients in intensive care unit. A report of thromboelastography findings and other parameters of hemostasis. J Thromb Haemost 2020 [Epub ahead of print].

6 Artifoni M, Danic G, Gautier G et al. Systematic assessment of venous thromboembolism in COVID-19 patients receiving thromboprophylaxis: incidence and role of D-dimer as predictive factors. J Thromb Thrombolysis 2020;50:211-6.

7 Wang T, Chen R, Liu C et al. Attention should be paid to venous thromboembolism prophylaxis in the management of COVID-19. Lancet Haematol 2020;7:e362-3. 